\title{
A Systematic Literature Review on Metaheuristic Optimization Techniques in WSNs
}

\author{
Omar Gouda, Ali Bou Nassif, Manar AbuTalib, Qassim Nasir \\ OpenUAE Research and Development Group \\ University Of Sharjah \\ Sharjah, United Arab Emirates \\ U19104867@sharjah.ac.ae, anassif@sharjah.ac.ae,mtalib@sharjah.ac.ae, nasir@sharjah.ac.ae
}

\begin{abstract}
Metaheuristic algorithms are recognized for developing new algorithms and optimizing various aspects in Wireless Sensor Networks (WSNs). Evaluating a multitude of possible modes is required, in most complicated problems, to obtain an exact solution. Metaheuristic algorithms can obtain solutions in acceptable time constraints. These algorithms play an operational role in solving such problems by optimizing the different metrics such as coverage rate and energy consumption of the networks. These metrics have valuable impact on network lifetime as well. This systematic review focuses on the published work from 2010 to 2020 in metaheuristic optimization in WSN. Furthermore, the systematic review will answer multiple questions that will be discussed in the methodology section.
\end{abstract}

Index Terms-Optimization, Systematic Literature Review (SLR), Wireless Sensor Network (WSN), Metaheuristic, Network lifetime, Node Localization, Clustering, Routing, Coverage.

\section{INTRODUCTION}

Wireless Sensor Network consists of a sensor field that composes of sensor nodes, base stations, radio receivers for the various multipurpose functions and applications. Each node comprises of memory, sensors, analog to digital converter, micro-controller transmitter all this enable to work in one unit as single node when power is supply to them. The purpose of this is for tracking, detecting, monitoring of various real-life application [1].

Although they are having many issues and limitations regarding their limited battery life because whole architecture is working using power only. If power supply is limited it is affected the performance of network that results into increase in the number of dead nodes. As the dead nodes increase, the performance of the network is affected and degraded the lifetime of network [2].

To serve the same objective many researchers provided efficient models for better performance of WSN. The applications of WSN are available in different fields such as in military, health sector, aviation sector and telecommunication sector. Therefore, it is necessary to analyze and study the performance of WSN on various different optimization algorithms [3].

Metaheuristic methods are an important class of solution methods for practical optimization problems in WSN exhibiting high computational complexity. These methods are intended to quickly provide near-optimal solutions to complex optimization problems that cannot be solved exactly. Their advantages include easy implementation, rapidly-obtained so- lutions and robustness to variations in problem characteristics [4].

The rest of this paper is comprised as follows: Related work is discussed in Section 2. Problem formulation and technical background are presented in Sections 3 and 4 respectively. Section 5 presents the methodology followed in this Systematic Literature Review (SLR). Finally, we conclude our research with results and discussions in Section 6 and conclusion in Section 7.

\section{RELATED WORK}

Some SLRs have been conducted in the area of optimization of WSN. Mahapatra et al [5] presented a review of WSN and its Quality of Service (QoS) parameters using Nature-Inspired (NA) Optimization Algorithms. The SLR focused on papers that are concerned with optimizing the parameters of QoS of the WSN. In addition, the paper specified the difference between the application specific QoS and network specific QoS. As the application specific takes factors particular to an application e.g. (area of coverage and number of active sensor nodes). On the other hand, the network specific QoS implies the efficient use of network resources like power usage. Also, the paper presented a brief review on the NA algorithms. The review did not follow any of the popular SLR structures. The overall summary of the review discussed the algorithms used, the impacted factors and the main focus of each paper. The review concluded with some challenges and future scope of for further research work.

Shafiq et al [6] conducted a review on energy efficient routing schemes in WSN. The paper followed the Kitchenham SLR scheme [7]. Furthermore, the review has categorized the algorithms into 8 categories, all concerned with energy efficient routing. Some of these categories are (Power efficient gathering, Energy efficient distributing and clustering and Secure energy efficient routing algorithms). The overall summary of the review had different criteria suitable for each category. This SLR is different from the above as it is based on the guidelines in PRISMA [8] and the key contribution are as follows:

1) A Systematic Literature Review is performed to analyze the existing latest schemes addressing several challenges in WSN. 
2) A number of related energy efficient schemes during 2010 to 2020 are searched as per research question and thoroughly studied.

3) Presents an overall review of four challenges in WSNs.

4) The different types of algorithms (hybrid, Modified or Standard) are showcased in this review.

\section{Problem Formulation}

In WSN, network resources are very limited and should be used effectively and efficiently. WSN characteristics were designed while having this limitation in mind like clustering. But this was not enough to prolong the lifetime or the high energy consumption of the nodes. Thus, these characteristics and challenges had to be solved or optimized. In this section, the characteristics and challenges addressed by this literature review will be explained and form the optimization problem [9].

\section{A. Clustering}

A cluster is a group of sensor nodes where one of the sensor nodes works as Cluster Head $(\mathrm{CH})$ and other nodes are called Cluster Members (CMs). The main responsibility of the $\mathrm{CH}$ is to collect the data from its CMs and transmit the aggregated data to the Base Station (BS) either directly or using multihop routing. $\mathrm{CH}$ near to the sink not only collects the data from its CMs but also works as a relay node for forwarding the packets of the other $\mathrm{CHs}$ towards sink node [10].

Each method used their own objective function, but generally the objective were minimizing the intra-cluster distance and energy of cluster head [11].

\section{B. Routing}

To achieve an energy efficient routing, most of the publications chose to minimize the number of nodes as well as minimizing the distance between the active nodes:

The $D\left(s_{i}, \rho\right)$ is the Euclidian Distance between the node $s i$ and point $\rho$ shown in eq. (1).

$$
\begin{aligned}
\min \left[D\left(s_{i}, \rho\right)=\right. & \left.\sqrt{\left(x_{i}-x\right)^{2}+\left(y_{i}-y\right)^{2}}\right] \\
& \min \left[\frac{R}{C}\right]
\end{aligned}
$$

Where $\mathrm{R}$ is the total number of relay nodes in the network. C is the total number of cluster head candidates that act as relay nodes.

\section{Coverage}

To measures how much area of Region of Interest (RoI) has been covered the concept of coverage ratio can be used. Coverage ratio is a measure of what percentage of area of RoI has been covered by nodes. The formula of coverage has been given in eq. (3).

$$
C_{R}=\frac{m}{n}
$$

Here, $m$ is the unique number of grid points insides the RoI which have been covered by the sensors and $\mathrm{n}$ is the total number of grid points inside the RoI [12].

\section{Node Localization}

To localize the node accurately, most of the methods try to minimize the location error [13]. Thus, mean squared error (MSE) is used as objective function between the actual and estimated distances of computed node coordinates and the actual node coordinates:

$$
M S E=\frac{1}{M} \sum_{i=1}^{M}\left(d_{i}-\hat{d}\right)^{2}
$$

Where $d_{i}$ is actual distance and $\hat{d}$ is estimated distance (the value $d_{i}$ obtained from noisy range measurements) and $M \geq 3$ (location of the sensor node needs a minimum of three anchors within transmission range $R$ ).

\section{TECHNICAL BACKGROUND}

Both old and new metaheuristic algorithms are still being used in several research areas. WSN had its share of these algorithms. Thus, a background about some of these algorithms is discussed in this section.

\section{A. Ant Colony Optimization (ACO)}

Ant Colony Optimization is a bio-inspired algorithm based on the behavior of real ants. It has been observed that a group of ants can cooperatively figure out the shortest route between their nest and their food. The communication between ants happens through volatile chemical substances known as pheromones [14].

\section{B. Artificial Bee Colony (ABC)}

It is an important bio-inspired algorithm inspired by the collective foraging behavior of honey bees [15]. Like ACO it also searches for an optimal numerical solution among large data sets. Through the social cooperation of three types of bees namely employed bees, onlooker bees, and scout bees they can complete their task. The employed bees take the responsibility of searching food around the food source and share the information with the onlooker bees and finally these types of bees select good food sources from those discovered by the employed bees. ABC can address the multi-dimensional numeric problem [16].

\section{Whale Optimization Algorithm (WOA)}

In recent days more and more modern metaheuristic algorithms are emerging. The Whale optimization algorithm is one of the most recently proposed metaheuristic optimization algorithms introduced by Mirjalili, S. and Lewis, A. in 2016 [17]. WOA mimics the hunting mechanism of humpback whales in nature. In this short period due to its easy implementation, this algorithm has been used in a wide range of problems in several domains including WSNs [18]

\section{Grey Wolf Optimization $(G W O)$}

A new metaheuristic called Grey Wolf Optimizer (GWO) inspired by grey wolves have been proposed by Mirjalili et al. in [19]. The GWO algorithm mimics the leadership hierarchy and hunting mechanism of grey wolves in nature [20]. 


\section{Methodology}

SLR is a type of literature reviews that helps to discover, categorize, and examining the existing literature for any specific research question. The main purpose of SLR is to evaluate the existing literature as per research question and find the gap. This SLR is following the guidelines provided by PRISMA [8].

\section{A. Research Questions}

The main goal of this systematic review is to identify and examine research papers that optimize WSN in different areas . Based on that, the following research questions are identified:

RQ1 What are the different types of papers that are included in the study?

RQ2 What are the optimization algorithms commonly used to solve each challenge?

RQ3 What are the most common challenge solved using the metaheuristic or hybrid algorithms?

RQ4 What are the algorithms used to solve each challenge?

RQ5 What are the objectives used for each proposed method?

RQ6 What are the other metaheuristic algorithms that are compared with each proposed method?

RQ7 What is the type of each algorithm (Hybrid, Modified or Standard)?

RQ8 What are the advantages/drawbacks of each method?

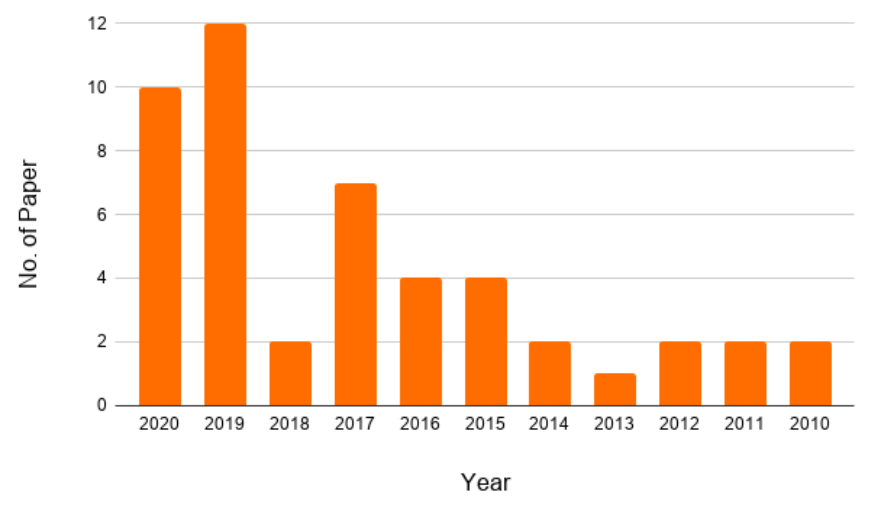

Fig. 1: Papers distribution over the years.

\section{B. Search Strategy}

1) Search Keywords: After defining research questions, the second most important step is to design strings and phases that help in searching. In order to have a quality research multiple steps are followed in this paper as mentioned below:

i Identifying the focal words and the complete understanding of the research question.

ii Use different replacements of phrases and words that can be used for literature searching

iii Combine different words to produce meaningful phrases.

iv Keywords used for researching: "Optimization, WSN, metaheuristic, Wireless Sensor Network, PSO, ACO, $\mathrm{ABC} "$
2) Research Resources: The following digital libraries were used to search for the needed research papers:

get real sources

- Google Scholar

- IEEE Xplore

- Science Direct

- ResearchGate

- Springer

\section{Screening and Selection}

Originally, 80 papers were collected which were obtained from the mentioned resources using the search keywords. Further filtration was done by the authors to ensure only the valid and relevant papers were included as show in Fig. 2. The filtration included:

1) Obtain the date of publish and remove any paper before 2010.

2) Read Abstract and conclusion of each paper and remove the irrelevant and duplicates

3) Read the full research paper and choose the relevant papers to the topic.

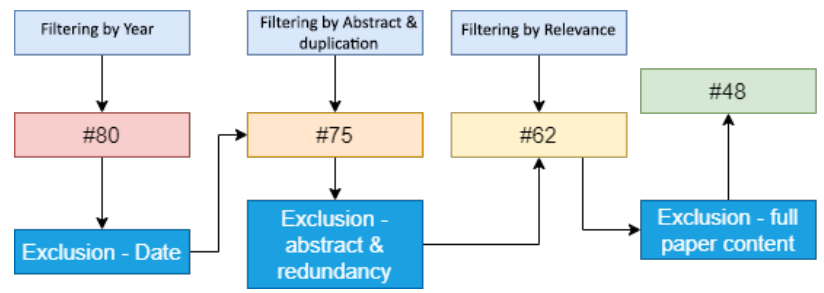

Fig. 2: Screening and Selection stages.

\section{Data Extraction Strategy}

In this stage, the finalized list of papers was used to extract needed information to answer the set of research questions. Paper title, Ref ID, publication year, publication type and answers for research questions 1 to 8. It is also important to note that not all papers answered all research questions.

\section{E. Synthesis Extracted Data}

The extracted information obtained from answering RQ5RQ8 in tabulated form and presented as comparison. while the other research questions were put as graphs and presented in results section.

\section{RESUlTS AND Discussions}

\section{A. Papers distribution (RQ1)}

The total papers that were included in this review are 48 papers. These papers fall in 2 main categories, conference papers and journal papers. The majority of these papers fall into the journal papers at $77 \%$. The rest $23 \%$ are conference papers. As shown in figure 1, most of the papers included were published in the past 5 years with $81 \%$ of the total number of papers. 
TABLE I: Analysis of collected papers for questions (4-8)

\begin{tabular}{|c|c|c|c|c|c|c|}
\hline Citation & Algorithm & Type & Challenge & $\begin{array}{l}\text { Algorithms } \\
\text { compared }\end{array}$ & Objective Func. & Other Advantages \\
\hline$[21], 2020$ & EHMO & Hybrid & Routing & PSO,ACO,DE,SA & Energy/Lifetime & Lifetime \\
\hline$[22], 2020$ & WOA-SA & Hybrid & Clustering & ABC,GA,AGSA,WOA & Intra-cluster/Energy & Energy reduction \\
\hline$[23], 2020$ & TLBO & Modified & Clustering & $\begin{array}{c}\text { PSO-C,GA- } \\
\text { based,PSO-SD }\end{array}$ & Delay and Energy & Lifetime \\
\hline [9],2020 & DA-FF & Hybrid & Clustering & GA,GWO,WOA,FF,DA & Energy & Lifetime \\
\hline$[24], 2020$ & $\mathrm{ABO}$ & Standard & Routing & - & Energy & Energy reduction \\
\hline$[26], 2020$ & HAGOA & Hybrid & Routing/Clustering & LEACH,PSO,ACO,ABC & $\begin{array}{c}\text { Intra-cluster /hop } \\
\text { count }\end{array}$ & Lifetime \\
\hline$[11], 2020$ & DEABC & Hybrid & Clustering & PSO-BSP,LEACH & Intra-cluster & Lifetime \\
\hline$[10], 2020$ & Memtic & Modified & Clustering & GA,LEACH & $\begin{array}{c}\text { Node } \\
\text { Degree/Intra-cluster }\end{array}$ & Energy reduction \\
\hline$[27], 2020$ & PSO & Modified & Coverage & PSO & Energy & - \\
\hline$[28], 2019$ & KHO & Modified & Routing & - & Distance/Delay & Energy reduction \\
\hline$[29], 2019$ & $\mathrm{ACO}$ & Standard & Clustering & - & Intra-cluster/energy & Energy reduction \\
\hline$[31], 2019$ & $\mathrm{CSO}$ & Standard & Routing & GWO,A3 & Intra-cluster/Energy & Energy reduction \\
\hline$[32], 2019$ & OB-CWO & Modified & Clustering & WOA,GSA,PSO & No. of cluster heads & Lifetime \\
\hline$[33], 2019$ & SKFO & Standard & Coverage & PSO,GA & energy/throughput & - \\
\hline$[34], 2019$ & DEA & Standard & Routing & GWO,BAT,ACO & Error in localization & Energy reduction \\
\hline$[35], 2019$ & WOA & Standard & Node localization & PSO,DE,GWO,MFOA & Error in localization & - \\
\hline$[36], 2019$ & $\mathrm{EHO}$ & Standard & Node localization & BBO,PSO, FFO & Error in localization & - \\
\hline$[37], 2019$ & FPO & Modified & Node localization & PSO, FPA, pFPA & $\begin{array}{c}\text { Node energy/Node } \\
\text { degree/Intra } \\
\text { clustering/Coverage }\end{array}$ & - \\
\hline$[38], 2019$ & ACO-PSO & Hybrid & Clustering & GSTEB, ACO & $\begin{array}{c}\text { Node } \\
\text { power/Lifetime }\end{array}$ & Lifetime \\
\hline$[39], 2019$ & $\mathrm{ABC}$ & Modified & Clustering & - & $\begin{array}{c}\text { Region Of } \\
\text { Interest/No. of } \\
\text { nodes }\end{array}$ & Lifetime \\
\hline$[40], 2018$ & ACO-PSO & Hybrid & Clustering & $\mathrm{GA}, \mathrm{ABC}$ & Error in localization & Lifetime \\
\hline$[13], 2018$ & $\mathrm{EHO}$ & Standard & Node localization & $\begin{array}{l}\text { PSO MSPSO ABC } \\
\text { MSABC }\end{array}$ & Intra-cluster/Energy & - \\
\hline$[42], 2017$ & GA & Standard & Routing & - & Region Of Interest & Energy reduction \\
\hline$[43], 2017$ & CS-HS & Hybrid & Routing/Clustering & $\begin{array}{c}\text { LEACH, } \\
\text { PSO-ECHS }\end{array}$ & Error in localization & Energy reduction \\
\hline$[44], 2017$ & GWO & Standard & Node localization & PSO MBA & Maximize coverage & - \\
\hline$[45], 2017$ & PSO-SA & Hybrid & Clustering & $\mathrm{CSA}, \mathrm{LEACH}-\mathrm{C}$ & Residual energy & Energy reduction \\
\hline$[46], 2017$ & TLBO & Standard & Routing & PSO, ACO and HS & $\begin{array}{c}\text { Distance Between } \\
\text { nodes, }\end{array}$ & Lifetime \\
\hline$[47], 2017$ & $\mathrm{BOA}$ & Standard & Node localization & PSO,FA & $\begin{array}{c}\text { Region Of } \\
\text { Interest/No. of } \\
\text { nodes }\end{array}$ & - \\
\hline$[48], 2016$ & FWA & Modified & Coverage & PSO & Intra-cluster/Energy & - \\
\hline$[49], 2016$ & HS-Kmean & Hybrid & Clustering & $\begin{array}{l}\text { K-means, HSA } \\
\text {,LEACH ,EAERP }\end{array}$ & Dissipated energy & Energy reduction \\
\hline$[50], 2016$ & $\mathrm{ABC}$ & Standard & Clustering & $\begin{array}{l}\text { PSO-HC,PSO- } \\
\text { C,LEACH- } \\
\text { C,LEACH }\end{array}$ & $\begin{array}{c}\text { distance between } \\
\text { nodes }\end{array}$ & Energy reduction \\
\hline$[51], 2016$ & GWO & Standard & Routing/Clustering & $\begin{array}{l}\text { SEP, DEEC, } \\
\text { LEACH }\end{array}$ & stability period & Energy reduction \\
\hline$[52], 2015$ & FOA-MAP & Hybrid & Node localization & $\begin{array}{l}\text { BOAMAP,MCS- } \\
\text { MAP }\end{array}$ & Intra-cluster/Energy & - \\
\hline$[53], 2015$ & $\mathrm{ACO}$ & Standard & Routing & - & Intra-cluster & Lifetime \\
\hline$[54], 2015$ & TCO & Standard & Coverage & & Error in localization & - \\
\hline$[55], 2015$ & PSO & Modified & Routing/Clustering & $\begin{array}{c}\text { LEACH,PSO-C,GA- } \\
\text { C,LEACH-C }\end{array}$ & $\begin{array}{c}\text { No. of relay } \\
\text { nodes/Worst quality } \\
\text { branch }\end{array}$ & Energy reduction \\
\hline$[56], 2014$ & HS & Standard & Clustering & LEACH-C, FCM & Error in localization & Lifetime \\
\hline$[64], 2014$ & $\mathrm{ACO}$ & Modified & Routing & $\mathrm{ACO}$ & Error in localization & Energy reduction \\
\hline$[57], 2013$ & $\mathrm{ACO}$ & Modified & Routing & LEACH & Region Of Interest & Lifetime \\
\hline$[58], 2012$ & PSO & Modified & Node localization & GA,SA, PSO & Intra-cluster/Energy & - \\
\hline$[59], 2012$ & BAT & Standard & Clustering & LEACH & $\begin{array}{c}\text { No. of relay } \\
\text { nodes/Worst quality } \\
\text { branch }\end{array}$ & Energy reduction \\
\hline$[60], 2011$ & EAERP & Hybrid & Routing & LEACH,SEP,HCR & Error in localization & Lifetime \\
\hline$[61], 2011$ & $\mathrm{ACO}$ & Standard & Coverage & $\mathrm{SA}, \mathrm{CHC}$ & No. of nodes & - \\
\hline
\end{tabular}




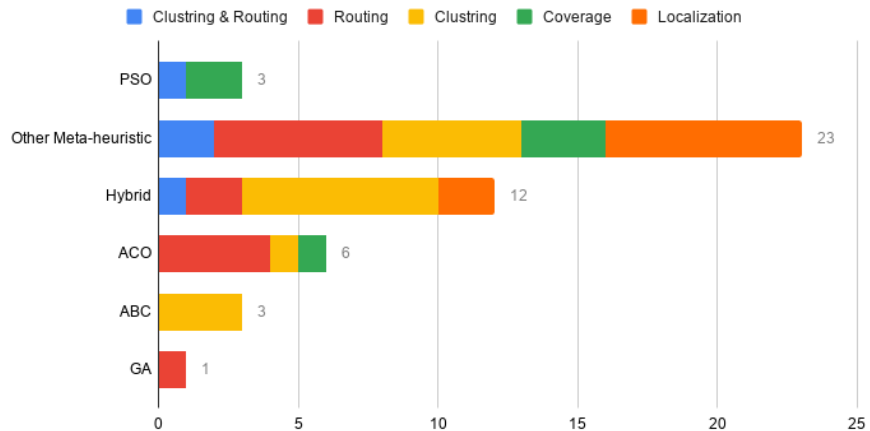

Fig. 3: Most common metaheuristic algorithms used to solve WSN Challenges.

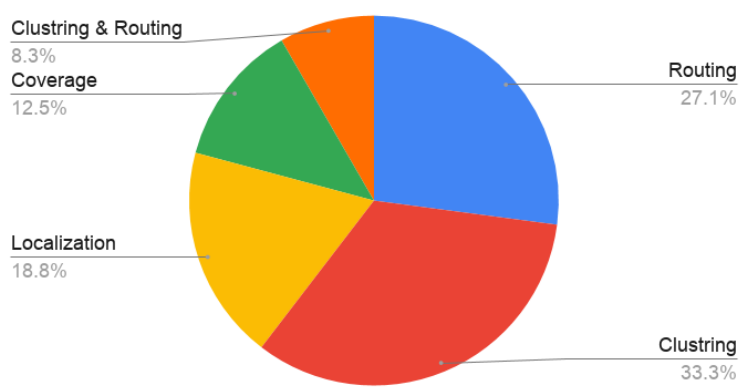

Fig. 4: Most common challenge solved using metaheuristic methods.

\section{B. Frequently used Algorithms (RQ2)}

Around 23 methods out of 48 were using new metaheuristic algorithms like BAT, BOA and TLBO algorithms. Followed by 12 papers using hybrid algorithms which combines the traditional metaheuristic logarithms together or with new methods as well. The rest 13 papers were using either the standard version of the traditional metaheuristic algorithms or with some modifications and improvements. These results can be seen in figure 3 . and table I.

\section{Commonly Addressed Challenges (RQ3)}

Clustering had the largest number of paper trying to optimize it with $33.3 \%$ followed by Routing with $27.1 \%$. The rest $39.6 \%$ are distributed over localization with $18.8 \%$ and coverage $12.5 \%$ and $8.3 \%$ for optimizing both clustering and routing with the same method. As shown in figure 4 .

\section{Research Question 4-8}

As shown in table I, the research paper were ordered descendingly based on the publication year. In this table, each research paper was identified with their algorithm used, type of algorithm, addressed challenge, objective function, algorithms compared and other advantages. This table helps in answering multiple research questions, more specifically research questions 4 to 8 .

\section{CONCLUSION}

WSN is getting more and more common, since the technology is increasing very quickly, and therefore sensors are being used in multiple environment. Even though sensor are commonly being used, they are considered to be very complex due to the limited amount of energy and memory they are able to use. This research is done on the exiting data. This survey discuss SLR performed in order to build research question and literature review. Many steps are performed in order to select research papers related to research question. A trend can be noticed from this review as many researches are using the same objective function with no significant contribution overall. Also, more challenges of WSN can still be explored using optimization. Most of the WSN challenges were not addressed using the met-heuristic nor optimization algorithms in general.

\section{REFERENCES}

[1] F. Losilla, A. -J. Garcia-Sanchez, F. Garcia-Sanchez, J. Garcia-Haro, and Z. J. Haas, "A Comprehensive Approach to WSN-Based ITS Applications: A Survey," Sensors, vol. 11, no. 11, pp. 10220-10265, Oct. 2011

[2] J. I. Huircán et al. , "ZigBee-based wireless sensor network localization for cattle monitoring in grazing fields," Computers and Electronics in Agriculture, vol. 74, no. 2, pp. 258-264, Nov. 2010, doi: 10. 1016/j. compag. 2010. 08. 014.

[3] F. V. C. Martins, E. G. Carrano, E. F. Wanner, R. H. C. Takahashi, and G. R. Mateus, "A Hybrid Multiobjective Evolutionary Approach for Improving the Performance of Wireless Sensor Networks," IEEE Sensors Journal, vol. 11, no. 3, pp. 545-554, Mar. 2011, doi: 10. 1109/jsen. 2010. 2048897.

[4] A. Gogu, D. Nace, A. Dilo, and N. Meratni, "Review of Optimization Problems in Wireless Sensor Networks," in Telecommunications Networks - Current Status and Future Trends, InTech, 2012.

[5] C. Mahapatra, A. Payal, and M. Chopra, "Review of WSN and Its Quality of Service Parameters Using Nature-Inspired Algorithm," in Advances in Intelligent Systems and Computing, Springer Singapore, 2019, pp. 451-461.

[6] M. Shafiq, H. Ashraf, A. Ullah, and S. Tahira, "Systematic Literature Review on Energy Efficient Routing Schemes in WSN - A Survey," Mobile Networks and Applications, Feb. 2020, doi: 10. 1007/s11036-020-01523-5.

[7] B. Kitchenham, O. Pearl Brereton, D. Budgen, M. Turner, J. Bailey, and S. Linkman, "Systematic literature reviews in software engineering - A systematic literature review," Information and Software Technology, vol. 51, no. 1, pp. 7-15, Jan. 2009, doi: 10. 1016/j. infsof. 2008. 09. 009.

[8] A. Liberati et al. , "The PRISMA statement for reporting systematic reviews and meta-analyses of studies that evaluate healthcare interventions: explanation and elaboration," BMJ, vol. 339, no. jul21 1, pp. b2700-b2700, Jul. 2009, doi: 10. 1136/bmj. b2700

[9] T. A. Alghamdi, "Energy efficient protocol in wireless sensor network: optimized cluster head selection model," Telecommunication Systems, Mar. 2020.

[10] V. K. Chawra and G. P. Gupta, "Load Balanced Node Clustering scheme using Improved Memetic Algorithm based metaheuristic Technique for Wireless Sensor Network," Procedia Computer Science, vol. 167, pp. 468-476, 2020, doi: 10. 1016/j. procs. 2020. 03. 256.

[11] G. P. Gupta and B. Saha, "Load balanced clustering scheme using hybrid metaheuristic technique for mobile sink based wireless sensor networks," Journal of Ambient Intelligence and Humanized Computing, Apr. 2020, doi: 10. 1007/s12652-020-01909-z.

[12] Y. Zou and Krishnendu Chakrabarty, "Sensor deployment and target localization based on virtual forces," in IEEE INFOCOM 2003. Twenty-second Annual Joint Conference of the IEEE Computer and Communications Societies (IEEE Cat. No. 03CH37428), 2003, doi: 10. 1109/infcom. 2003. 1208965.

[13] I. Strumberger, M. Beko, M. Tuba, M. Minovic, and N. Bacanin, "Elephan Herding Optimization Algorithm for Wireless Sensor Network Localization Problem," in IFIP Advances in Information and Communication Technology, Springer International Publishing, 2018, pp. 175-184.

[14] Dorigo, M. , Birattari, M. , Blum, C. , Gambardella, L. M , Mondada, F. and Stützle, T. , 2004. Ant Colony Optimization and Swarm Intelligence: 4th International Workshop, ANTS 2004. Proceedings. Lecture Notes in Computer Science, 3172.

[15] D. Karaboga, "Artificial bee colony algorithm," Scholarpedia, vol. 5, no. 3, p. $6915,2010$.

[16] Y. Yue, L. Cao, and Z. Luo, "Hybrid Artificial Bee Colony Algorithm for Improving the Coverage and Connectivity of Wireless Sensor Networks," Wireless Personal Communications, vol. 108, no. 3, pp. 1719-1732, May 2019.

[17] Mirjalili S, Lewis A (2016) The whale optimization algorithm. Adv Eng Softw 95:51-67

[18] El Aziz MA, Ewees AA, Hassanien AE (2017) Whale optimization algorithm andmoth-flame optimization for multilevel thresholding image segmentation. Expert Syst Appl 83:242-256

[19] Mirjalili S, Mirjalili SM, Lewis A (2014) Grey wolf optimizer. Adv Eng Softw 69:46-61

[20] Kohli M, Arora S (2018) Chaotic grey wolf optimization algorithm for constrained 
[21] H. Wang, K. Li, and W. Pedrycz, "An Elite Hybrid Metaheuristic Optimization Algorithm for Maximizing Wireless Sensor Networks Lifetime With a Sink Node," IEEE Sensors Journal, vol. 20, no. 10, pp. 5634-5649, May 2020.

[22] C. Iwendi, P. K. R. Maddikunta, T. R. Gadekallu, K. Lakshmanna, A. K. Bashir, and M. J. Piran, "A metaheuristic optimization approach for energy efficiency in the IoT networks," Software: Practice and Experience, Feb. 2020, doi: 10. 1002/spe. 2797.

[23] M. Masdari and S. Barshandeh, "Discrete teaching-learning-based optimization algorithm for clustering in wireless sensor networks," Journal of Ambient Intelligence and Humanized Computing, Mar. 2020, doi: 10. 1007/s12652-020-01902-6.

[24] J. B. Odili, M. N. M. Kahar, and S. Anwar, "African Buffalo Optimization: A Swarm-Intelligence Technique," Procedia Computer Science, vol. 76, pp. 443-448, 2015.

[25] A. Mishra, S. Choudhary, M. Vats, and S. Sachan, "LEACH with Pheromone Energy Efficient Routing in Wireless Sensor Network," in Intelligent Computing in Engineering, Springer Singapore, 2020, pp. 91-98.

[26] B. P. Dahiya, S. Rani, and P. Singh, "Lifetime Improvement in Wireless Sensor Networks Using Hybrid Grasshopper metaheuristic," in Lecture Notes in Electrical Engineering, Springer International Publishing, 2019, pp. 305-320.

[27] A. Anurag, R. Priyadarshi, A. Goel, and B. Gupta, "2-D Coverage Optimization In WSN Using A Novel Variant Of Particle Swarm Optimisation," in 2020 7th International Conference on Signal Processing and Integrated Networks (SPIN), 2020, doi: 10. 1109/spin48934. 2020. 9070978 .

[28] P. Visu, T. S. Praba, N. Sivakumar, R. Srinivasan, and T. Sethukarasi, "Bio-inspired dual cluster heads optimized routing algorithm for wireless sensor networks," Journal of Ambient Intelligence and Humanized Computing, Jan. 2020, doi: 10 1007/s12652-019-01657-9.

[29] K. Guleria and A. K. Verma, "metaheuristic Ant Colony Optimization Based Unequal Clustering for Wireless Sensor Network," Wireless Personal Communications, vol. 105 , no. 3, pp. 891-911, Feb. 2019

[30] H. T. T. Binh, N. T. Hanh, L. Van Quan, N. D. Nghia, and N. Dey, "Metaheuristics for maximization of obstacles constrained area coverage in heterogeneous wireless sensor networks," Applied Soft Computing, vol. 86, p. 105939, Jan. 2020, doi: 10 1016/j. asoc. 2019. 105939.

[31] M. M. Fouad, A. I. Hafez, and A. E. Hassanien, "Optimizing topologies in wireless sensor networks: A comparative analysis between the Grey Wolves and the Chicken Swarm Optimization algorithms," Computer Networks, vol. 163, p. 106882, Nov. 2019 .

[32] M. Kumar and A. Chaparala, "OBC-WOA: Opposition-Based Chaotic Whale Optimization Algorithm for Energy Efficient Clustering in Wireless Sensor Network," International Journal of Intelligent Engineering and Systems, vol. 12, no. 6, pp. 249-258, Dec. 2019, doi: 10. 22266/ijies2019. 1231. 24

[33] N. A. A. Aziz, Z. Ibrahim, N. H. A. Aziz, and K. A. Aziz, "Simulated Kalman Filter Optimization Algorithm for Maximization of Wireless Sensor Networks Coverage," in 2019 International Conference on Computer and Information Sciences (ICCIS), 2019, doi: 10. 1109/iccisci. 2019. 8716387.

[34] M. Masood, M. M. Fouad, S. Seyedzadeh, and I. Glesk, "Energy Efficient Software Defined Networking Algorithm for Wireless Sensor Networks," Transportation Research Procedia, vol. 40, pp. 1481-1488, 2019, doi: 10. 1016/j. trpro. 2019. 07. 205 .

[35] L. Baidar, A. Rahmoun, P. Lorenz, and M. Mihoubi, "Whale Optimization Approach for Optimization Problem In Distributed Wireless Sensor Network," in Proceedings of the 9th International Conference on Information Systems and Technologies - icist 2019, 2019, doi: 10. 1145/3361570. 3361594.

[36] I. Strumberger, M. Minovic, M. Tuba, and N. Bacanin, "Performance of Elephant Herding Optimization and Tree Growth Algorithm Adapted for Node Localization in Wireless Sensor Networks," Sensors, vol. 19, no. 11, p. 2515, Jun. 2019.

[37] T. -T. Nguyen, J. -S. Pan, and T. -K. Dao, "An Improved Flower Pollination Algorithm for Optimizing Layouts of Nodes in Wireless Sensor Network," IEEE Access, vol. 7, pp. 75985-75998, 2019

[38] N. Shivaraman and S. Mohan, "A Reactive Hybrid Metaheuristic Energy-Efficient Algorithm for Wireless Sensor Networks," in Smart Network Inspired Paradigm and Approaches in IoT Applications, Springer Singapore, 2019, pp. 1-13.

[39] S. Famila and A. Jawahar, "Improved Artificial Bee Colony Optimization-Based Clustering Technique for WSNs," Wireless Personal Communications, vol. 110, no. 4, pp. 2195-2212, Oct. 2019, doi: 10. 1007/s11277-019-06837-6.

[40] S. Kaur and R. Mahajan, "Hybrid metaheuristic optimization based energy efficient protocol for wireless sensor networks," Egyptian Informatics Journal, vol. 19, no. 3, pp. 145-150, Nov. 2018, doi: 10. 1016/j. eij. 2018. 01. 002.

[41] P. S. Mann and S. Singh, "Improved artificial bee colony metaheuristic for energyefficient clustering in wireless sensor networks," Artificial Intelligence Review, vol. 51, no. 3, pp. 329-354, May 2017, doi: 10. 1007/s10462-017-9564-4.

[42] P. S. Ragavan and K. Ramasamy, "Optimized routing in wireless sensor networks by establishing dynamic topologies based on genetic algorithm," Cluster Computing, vol. 22, no. S5, pp. 12119-12125, Jan. 2018.

[43] G. P. Gupta and S. Jha, "Integrated clustering and routing protocol for wireless sensor networks using Cuckoo and Harmony Search based metaheuristic techniques," Engineering Applications of Artificial Intelligence, vol. 68, pp. 101-109, Feb. 2018, doi: 10. 1016/j. engappai. 2017. 11. 003 .

[44] R. Rajakumar, J. Amudhavel, P. Dhavachelvan, and T. Vengattaraman, "GWOLPWSN: Grey Wolf Optimization Algorithm for Node Localization Problem in Wireless Sensor Networks," Journal of Computer Networks and Communications, vol. 2017, pp. 1-10, 2017, doi: 10. 1155/2017/7348141.
[45] M. T. Mekonnen and K. N. Rao, "Cluster Optimization Based on Metaheuristic Algorithms in Wireless Sensor Networks," Wireless Personal Communications, vol. 97, no. 2, pp. 2633-2647, Jul. 2017, doi: 10. 1007/s11277-017-4627-z.

[46] A. El Ghazi and B. Ahiod, "Energy efficient teaching-learning-based optimization for the discrete routing problem in wireless sensor networks," Applied Intelligence, vol. 48, no. 9, pp. 2755-2769, Dec. 2017, doi: 10. 1007/s10489-017-1108-8.

[47] S. Arora and S. Singh, "Node Localization in Wireless Sensor Networks Using Butterfly Optimization Algorithm," Arabian Journal for Science and Engineering, vol. 42, no. 8, pp. 3325-3335, Mar. 2017, doi: 10. 1007/s13369-017-2471-9.

[48] E. Tuba, M. Tuba, and D. Simian, "Wireless sensor network coverage problem using modified fireworks algorithm," in 2016 International Wireless Communications and Mobile Computing Conference (IWCMC), 2016.

[49] D. Raval, G. Raval, and S. Valiveti, "Optimization of clustering process for WSN with hybrid harmony search and K-means algorithm," in 2016 International Conference on Recent Trends in Information Technology (ICRTIT), 2016, doi: 10. 1109/icrtit. 2016. 7569584 .

[50] A. A. Abba Ari, A. Gueroui, B. O. Yenke, and N. Labraoui, "Energy efficient clustering algorithm for Wireless Sensor Networks using the ABC metaheuristic,' in 2016 International Conference on Computer Communication and Informatics (ICCCI), 2016, doi: 10. 1109/iccci. 2016. 7480010.

[51] N. A. Al-Aboody and H. S. Al-Raweshidy, "Grey wolf optimization-based energyefficient routing protocol for heterogeneous wireless sensor networks," in 2016 4th International Symposium on Computational and Business Intelligence (ISCBI), 2016, doi: 10. 1109/iscbi. 2016. 7743266.

[52] S. Sivakumar and R. Venkatesan, "metaheuristic approaches for minimizing error in localization of wireless sensor networks," Applied Soft Computing, vol. 36, pp. 506-518, Nov. 2015, doi: 10. 1016/j. asoc. 2015. 05. 053.

[53] R. Arya and S. C. Sharma, "Optimization approach for energy minimization and bandwidth estimation of WSN for data centric protocols," International Journal of System Assurance Engineering and Management, vol. 9, no. 1, pp. 2-11, Nov 2015 .

[54] P. P. Das, N. Chakraborty, and S. M. Allayear, "Optimal coverage of Wireless Sensor Network using Termite Colony Optimization Algorithm," in 2015 International Conference on Electrical Engineering and Information Communication Technology (ICEEICT), 2015.

[55] R. S. Y. Elhabyan and M. C. E. Yagoub, "Two-tier particle swarm optimization protocol for clustering and routing in wireless sensor network," Journal of Network and Computer Applications, vol. 52, pp. 116-128, Jun. 2015.

[56] D. C. Hoang, P. Yadav, R. Kumar, and S. K. Panda, "Real-Time Implementation of a Harmony Search Algorithm-Based Clustering Protocol for Energy-Efficient Wireless Sensor Networks," IEEE Transactions on Industrial Informatics, vol. 10, no. 1, pp. 774-783, Feb. 2014.

[57] Pratap Singh, M. , \& Gupta Amity, K. (2013). Techniques of Power Optimization for Wireless Sensor Network. International Journal of Computer Applications, 66(3), 975-8887.

[58] A. Kumar, A. Khosla, J. S. Saini, and S. Singh, "metaheuristic range based node localization algorithm for Wireless Sensor Networks," in 2012 International Conference on Localization and GNSS, 2012.

[59] Sharawi, M. , Emary, E. , Saroit, I. A. , \& El-mahdy, H. (2014). Bat Swarm Algorithm for Wireless Sensor Networks Lifetime Optimization. International Journal, 3(5), 654-664.

[60] E. A. Khalil and B. A. Attea, "Energy-aware evolutionary routing protocol for dynamic clustering of wireless sensor networks," Swarm and Evolutionary Computation, vol. 1, no. 4, pp. 195-203, Dec. 2011, doi: 10. 1016/j. swevo. 2011. 06.004.

[61] J. Tian, M. Gao, and G. Ge, "Wireless sensor network node optimal coverage based on improved genetic algorithm and binary ant colony algorithm," EURASIP Journal on Wireless Communications and Networking, vol. 2016, no. 1, Apr. 2016.

[62] G. Molina and E. Alba, "Location discovery in Wireless Sensor Networks using metaheuristics," Applied Soft Computing, vol. 11, no. 1, pp. 1223-1240, Jan. 2011, doi: 10. 1016/j. asoc. 2010. 02. 021.

[63] S. Okdem and D. Karaboga, "Routing in Wireless Sensor Networks Using Ant Colony Optimization," in First NASA/ESA Conference on Adaptive Hardware and Systems (AHS'06), 2010.

[64] A. El Ghazi, B. Ahiod, and A. Ouaarab, "Improved Ant Colony Optimization Routing Protocol for Wireless Sensor Networks," in Networked Systems, Springer International Publishing, 2014, pp. 246-256.

\section{AUTHORS CONTRIBUTIONS}

Omar Gouda wrote the paper. Ali Bou Nassif, Manar AbuTalib and Qassim Nasir conducted the methodology and revised the paper.

Creative Commons Attribution License 4.0 (Attribution 4.0 International, CC BY 4.0)

This article is published under the terms of the Creative Commons Attribution License 4.0 https://creativecommons.org/licenses/by/4.0/deed.en_US 